\title{
Turbulent slurry flow measurement using ultrasonic Doppler method in rectangular pipe
}

\author{
V. Bareš ${ }^{1, a}$, J. Krupička ${ }^{1}$, T. Picek ${ }^{1}, J$. Brabec ${ }^{1}$ and V. Matoušek ${ }^{1}$ \\ ${ }^{1}$ Department of Hydraulics and Hydrology, Czech Technical University in Prague, 16629 Prague 6
}

\begin{abstract}
Distribution of velocity and Reynolds stress was measured using ultrasonic velocimetry in flows of water and Newtonian water-ballotini slurries in a pressurized Plexiglas pipe. Profiles of the measured parameters were sensed in the vertical plane at the centreline of a rectangular cross section of the pipe. Reference measurements in clear water produced expected symmetrical velocity profiles the shape of which was affected by secondary currents developed in the rectangular pipe. Slurry-flow experiments provided information on an effect of the concentration of solid grains on the internal structure of the flow. Strong attenuation of velocity fluctuations caused by a presence of grains was identified. The attenuation increased with the increasing local concentration of the grains.
\end{abstract}

\section{Introduction}

Flow of slurry (i.e. mixture of water and solid grains at high concentration) occurs in many industrial applications and environmental processes. Turbulent slurry flow can be associated with various physical processes as erosion, suspension, transport, flocculation, settlement and consolidation the mechanisms of which remain poorly understood. One of the reasons is the difficulty in obtaining accurate measurements of the processes in a controlled environment [1].

High-concentrated slurry flows are studied in natural channels [1-4], laboratory flumes [5-6], and pressurized pipes [7-9]. An internal structure of slurry flow is one of the key issues in research on the slurry flow behaviour. The high volumetric concentration of grains in flowing slurry makes measurements of distribution of solids velocity and concentration a complex task and disqualifies techniques as Laser Doppler anemometry or Particle Image Velocimetry. The principal limitation of optical methods as used to date is that they can operate exclusively in clear fluids which allow photographic or video images to be recorded. This precludes its use on opaque materials such as slurries.

Acoustic methods may be a suitable option when it comes to measurement of a distribution of velocity in flowing slurries. The methods are capable of measuring an instantaneous velocity profile along the measuring line and they are applicable to opaque liquids and opaque pipe wall materials. Furthermore, the acoustic method applications can be non-intrusive, which is of vital importance in slurry flow testing.

\footnotetext{
${ }^{\mathrm{a}}$ Corresponding author: bares@fsv.cvut.cz
}

Recently, velocity profiles were successfully measured using ultrasonic velocimetry in pressurized pipes carrying non-Newtonian slurries composed of very fine grains at high volumetric concentration [10-12]. The question arises whether the method can be used with success also in pipes that transport Newtonian slurries composed of relatively coarse grains. This paper reports on our tests with such Newtonian slurries in the turbulent flow regime. Narrow-graded fractions of ballotini (glass beads) were used as model grains. Slurries of a broad range of solids concentrations were tested in our laboratory loop.

\section{Methods}

\subsection{Experimental loop}

Slurry-flow experiments were carried out in a closed pipe loop (Figure 1) in the Water Engineering Laboratory of the Czech Technical University in Prague. The loop is composed of horizontal- and vertical pipes. One part of the loop is the horizontal Plexiglas pipe of the length of 6 $\mathrm{m}$ and a rectangular cross section (inner width $50.8 \mathrm{~mm}$, inner height $51.2 \mathrm{~mm}$ ). The differential pressure is measured over Section 3 in this horizontal pipe determines the hydraulic gradient and hence the frictional pressure drop in slurry flow. Differential pressure is measured also in the vertical upgoing pipe (Section 1) and in the vertical downcoming pipe (Section 2) in order to determine the density of flowing slurry (and hence the average concentration of grains in the mixture). The flow 
rate is measured using an electromagnetic flow meter Krohne Optiflux 5000 installed at the vertical pipe in front of Section 1. A centrifugal pump driven by an electric motor maintains flow of slurry through the loop. The speed of the pump is smoothly regulated by a frequency converter. The temperature of liquid carrying grains is sensed in the loop in order to determine its viscosity and density. Data from all differential pressure transmitters, the flow meter and the thermometer are collected by an electronic data acquisition system. The thickness of sediment deposits in the horizontal transparent Plexiglas section is measured manually.

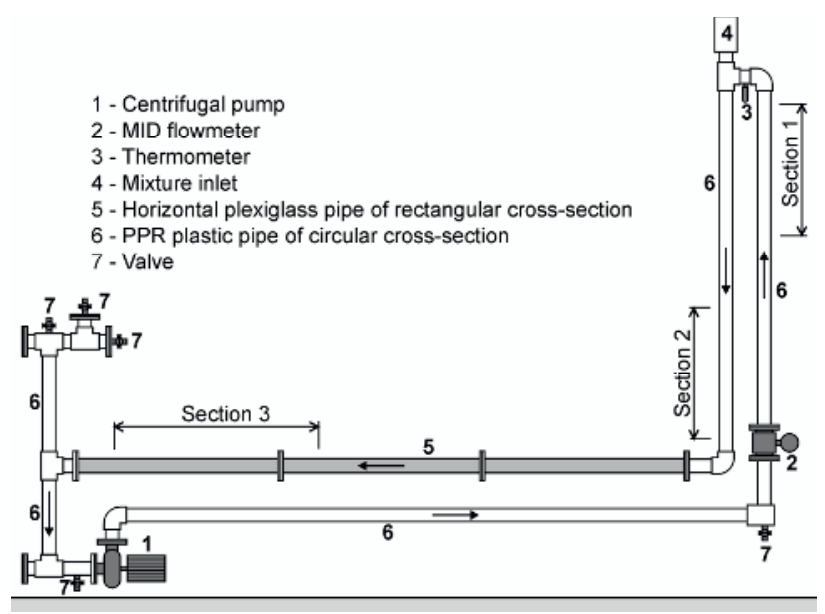

Figure 1. Scheme of experimental loop.

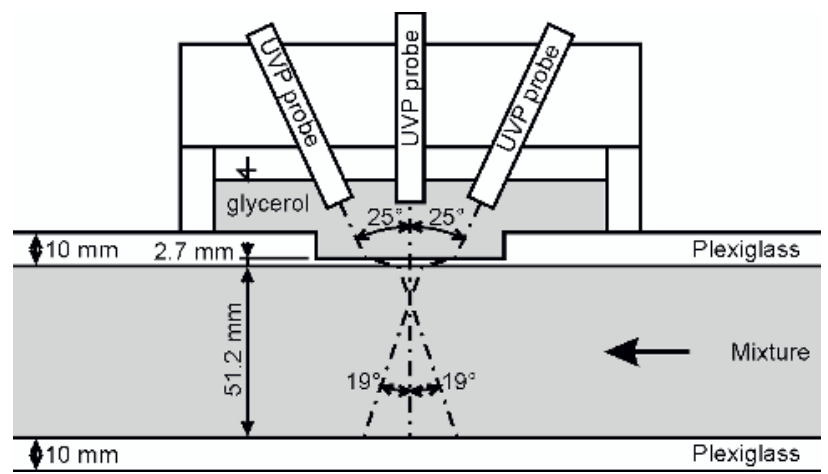

Figure 2. Arrangement of US transducers on Plexiglas pipe of rectangular cross section.

\subsection{Velocity measurements}

In Section 3 of the horizontal Plexiglas pipe, instantaneous velocity profiles were measured using Ultrasonic Velocity Profile (UVP) monitor (Met-Flow SA). UVP probes were located to the vertical longitudinal plane passing through the centre of the cross section of the rectangular pipe. The US transducers of basic frequencies of $4 \mathrm{MHz}, 2 \mathrm{MHz}$ and $1 \mathrm{MHz}$ were used depending on the concentration of grains and the velocity slurry flow. Lower basic frequencies were used for higher concentrations of grains. The sampling frequency was kept at constant level of $20 \mathrm{~Hz}(\mathrm{~d} t=50 \mathrm{~ms})$ for all experiments and the full range of basic frequencies.
Three different positions of the UVP probes were used (Figure 2). The angle of the transducers to the main flow normal (transducer 1 and 3) was 25 degree. However, the resulting active angle was estimated to 19 degree due to different acoustic impedance of glycerol and water. The velocity was decomposed using the assumptions postulated in previous studies [13-14]. Hence, the point time-averaged quantities of the longitudinal- and the vertical velocity and the Reynolds stress can be expressed as follows:

$$
\begin{aligned}
\bar{u} & =\frac{\overline{V_{R 1}}-\overline{V_{R 3}}}{2 \sin \alpha} \\
\bar{v} & =\frac{\overline{V_{R 1}}+\overline{V_{R 3}}}{2 \cos \alpha} \\
-\overline{u^{\prime} v^{\prime}} & =-\frac{\overline{V_{R 1}^{\prime}}-\overline{V_{R 3}^{\prime}}}{2 \sin 2 \alpha}
\end{aligned}
$$

where $V_{\mathrm{R}}$ are radial components measured by the individual transducers $1-3$.

\subsection{Test conditions}

A wide range of flow conditions was simulated in the experimental set-up. The average velocity of slurry in the pipe cross section varied from 0.2 to $3.5 \mathrm{~m} \cdot \mathrm{s}^{-1}$. Reynolds numbers Re were between $1.6 \cdot 10^{4}-1.5 \cdot 10^{5}$. The average volumetric concentration of grains (the ratio of volume of grains and the total volume of slurry in the loop) varied from 5 to $40 \%$. The delivered concentration of grains (the ratio of flow rate of grains and the total flow rate of slurry) varied from 3 to $30 \%$. The installed flow conditions (flow rate and average concentration) determined the mode of slurry flow - flow with stationary (upper plane) bed (UPB), stratified flow (SF) and heterogeneous flow (HF). In the UPB mode, the stationary deposit develops in the pipe; its top is eroded causing intense transport of grains above the deposit. The nature of SF flows is similar as they are significantly stratified, however there is no deposit - all particles are in motion. HF flow is much less stratified (Figure 3).
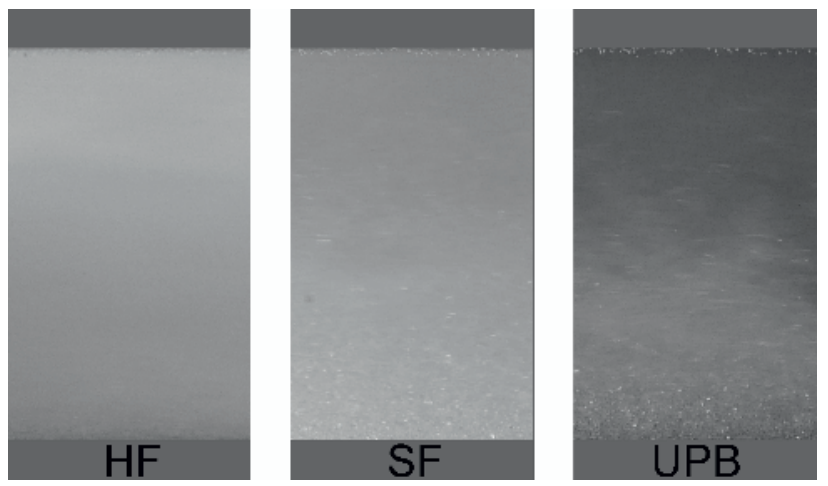

Figure 3. Visual comparison of studied transport regimes. 
The tested granular materials were three narrow graded fractions of industrial ballotini produced by PRECIOSA: B134, B9, and B7. Characteristic grain sizes are given in Table 1 . The density of grains was similar to natural sands and gravels $\left(2450 \mathrm{~kg} \cdot \mathrm{m}^{-3}\right)$. Our tests determined the settling velocity of the grains, $\mathrm{w}_{\mathrm{t}}$, to be 20 $\mathrm{m} \cdot \mathrm{s}^{-1}, 54 \mathrm{~m} \cdot \mathrm{s}^{-1}$, and $77 \mathrm{~m} \cdot \mathrm{s}^{-1}$. Hence, the grain Reynolds number was $\operatorname{Re}_{\mathrm{p}} \approx 3.5,23$, and 44 for the ballotini fraction B134, B9, and B7, respectively.

Table 1. Characteristic gain sizes in millimetres.

\begin{tabular}{|c|c|c|c|}
\hline & $\mathrm{d}_{50}$ & $\mathrm{~d}_{16}$ & $\mathrm{~d}_{84}$ \\
\hline B134 & 0.18 & 0.16 & 0.22 \\
\hline B9 & 0.42 & 0.34 & 0.48 \\
\hline B7 & 0.57 & 0.45 & 0.67 \\
\hline
\end{tabular}

\section{Results}

\subsection{Clear water flows}

\subsubsection{Velocity components}

As a reference, measurements of turbulent flows of clear water were carried out first. They delivered a vertical distribution of velocity components and turbulence characteristics in water flows. All experiments were carried out in the hydraulically-smooth regime with $\mathrm{Re}=$ $1.6 \cdot 10^{4}-1.0 \cdot 10^{5}$. Time-averaged longitudinal velocity profiles for different flow conditions are shown in Figure 4. As anticipated, symmetrical distributions with maxima in the centre of the rectangular duct were detected. Figure 5 normalizes the velocity profiles shown in Figure 4. There is an increase in the normalized maximum velocity with the increasing flow rate and Reynolds number.

The measured shape of the velocity profile is compared with the predicted shapes using different theoretical models and the experimental data by Launder and Ying [15] in Figure 6. Our experimental data exhibit higher maximum velocity in the pipe centre than the models and the other experimental data.

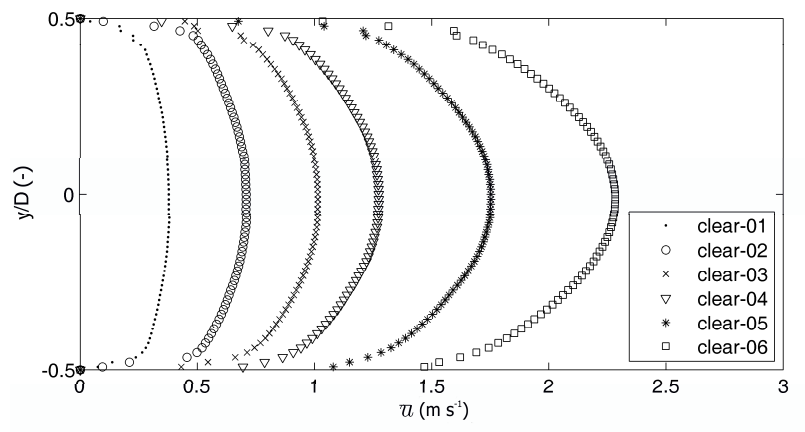

Figure 4. Vertical distribution of time-averaged longitudinal component of velocity of water using $4 \mathrm{MHz}$ transducer.

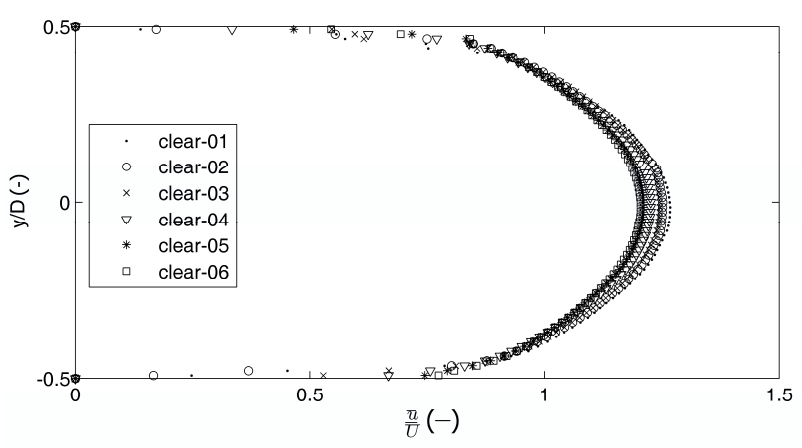

Figure 5. Normalized vertical distribution of time-averaged longitudinal component of velocity of water.

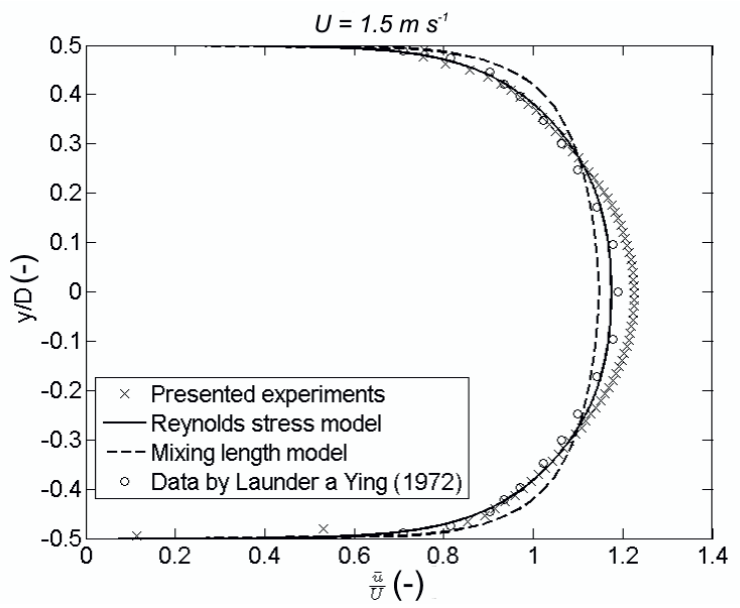

Figure 6. Comparison of measured profile of time-averaged longitudinal velocity with numerical simulations (mixing length model and Reynolds stress model) for average velocity $U=1.5$ $\mathrm{m} \cdot \mathrm{s}^{-1}\left(\operatorname{Re}=6 \cdot 10^{4}\right)$. Data of Launder and Ying for $\mathrm{Re}=2.2$. $10^{5}$.

A presence of vertical velocity components oriented from walls to the centre of the rectangular pipe can explain the differences. Our measurements detected the transversal velocities (see Figure 7) which correspond well with the findings of Knight and Patel [16] who described secondary currents in rectangular ducts. The mixing length model does not take secondary currents into account and that is why the predicted profile produces so small relative velocity in the pipe centre.

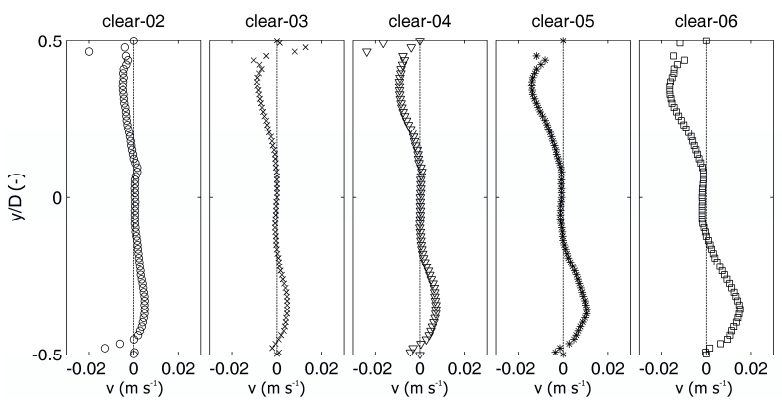

Figure 7. Vertical distribution of time-averaged vertical component of velocity of water. Water flows from walls to pipe centre along pipe centreline. 


\subsubsection{Reynolds stress distribution}

In theory, the normalized vertical distribution of Reynolds stress is a linear function of the flow depth with the maximum $( \pm 1.0)$ near the pipe wall and the zero value at the centerline. Figure 8 presents results of our measurements for different water-flow conditions. The wall shear tends to be of about $50 \%$ of the shear given by the energy grade line $\mathrm{i}_{\mathrm{e}}$ and the hydraulic radius $R$ as $u^{*^{2}}$ $=g \cdot R \cdot i_{\mathrm{e}}$. Such a difference can hardly be explained by secondary currents as the vertical velocity components are only of about $1 \%$ of the average velocity in the pipe cross section. Knight and Patel [16] reported maximum decrease of the Reynolds stress to be $10 \%$ compared to the averaged shear rate along the wetted perimeter of the pipe cross section.

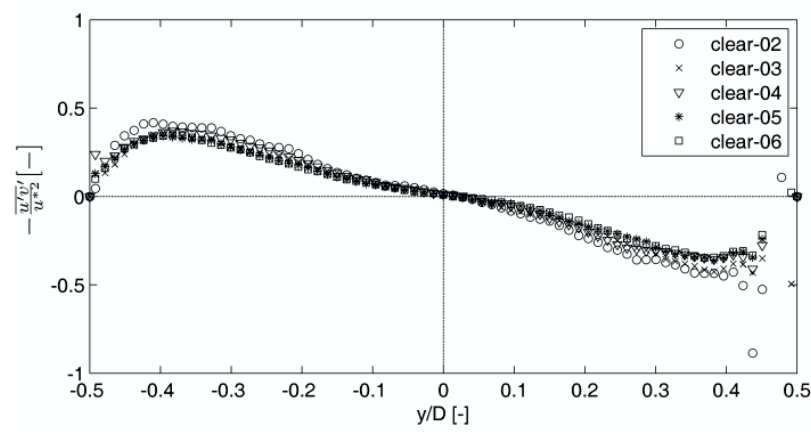

Figure 8. Normalized Reynolds stress distribution for $\mathrm{Re}=2.9$. $10^{4}-1.0 \cdot 10^{5}$.

\subsection{Slurry flows}

We present only results obtained in SF and HF transport modes. Typical distributions of the normalized velocity and the Reynolds stress are presented in Figure 9 (the SF mode) and Figure 10 (the HF mode) for slurry of ballotini B134.

In the SF mode, the velocity distribution is affected by the changing (increasing) local concentration along the vertical. The vertical position of the maximum local velocity shifted from the pipe centre to the upper part of the pipe due to the presence of a concentration profile in the pipe cross section. The higher local concentration of grains in the lower part of the cross section causes higher friction and deformation of a velocity profile. The higher concentration gradient the stronger asymmetry of the velocity profile.

The measured velocity distribution corresponds well with the Reynolds stress distribution. The maximum local velocity and the zero Reynolds stress occur both at the same vertical position $y / D$. The maximum values of the Reynolds stress are detected near the top wall of the pipe and near the top of the bed.

When the concentration distribution becomes virtually uniform (HT mode at high average velocity $U$ of flow), the velocity distribution becomes symmetrical. This affects also the Reynolds stress distribution; the zero value shifts to the centre of the rectangular pipe cross section (Figure 10).
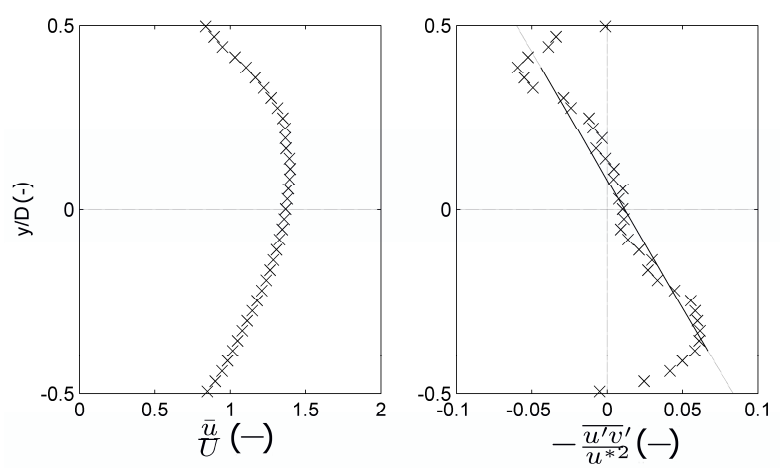

Figure 9. Ballotini B134 - distribution of normalized velocity (left) and Reynolds stress (right) for SF mode with delivered concentration of $24 \%$ and $U=1.7 \mathrm{~m} \cdot \mathrm{s}^{-1}\left(\operatorname{Re}=9.8 \cdot 10^{4}\right)$ using $2 \mathrm{MHz}$ transducer.
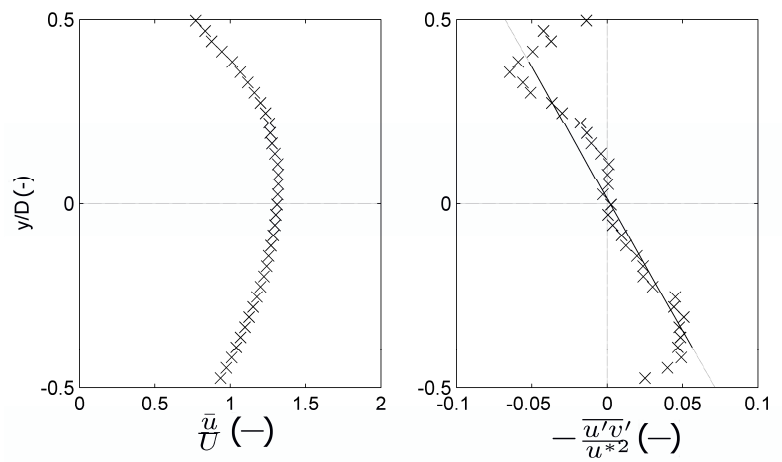

Figure 10. Ballotini B134 - distribution of normalized velocity (left) and Reynolds stress (right) for flow approaching HF mode with delivered concentration of $24 \%$ and $U=2.3 \mathrm{~m} \cdot \mathrm{s}^{-1}(\mathrm{Re}=$ $1.5 \cdot 10^{5}$ ) using $2 \mathrm{MHz}$ transducer.

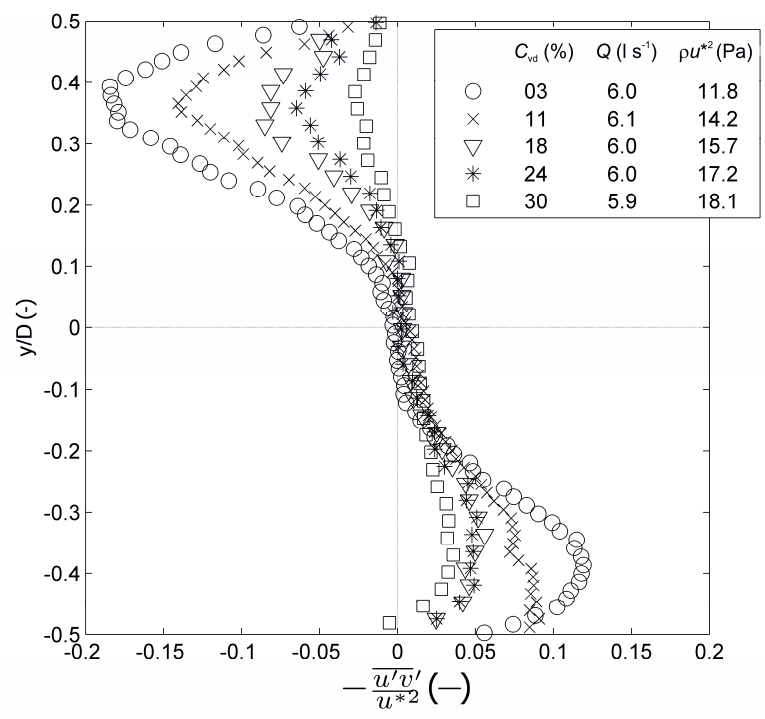

Figure 11. B134 - distribution of normalized Reynolds stress for flows with delivered concentrations from 3 to $30 \%$ and $U$ $2.3 \mathrm{~m} \cdot \mathrm{s}^{-1}\left(\operatorname{Re} \sim 1.5 \cdot 10^{5}\right)$ using 1 and $2 \mathrm{MHz}$ transducers.

In slurry flows, the measured instantaneous velocity is the velocity of transported grains (ballotini), not water. Velocity fluctuation of the grains seems to be strongly attenuated in flow of slurry even at small delivered concentration $C_{\mathrm{vd}}$ (Figure 11). A comparison of the slurry flow with the lowest concentration $\left(C_{\mathrm{vd}}=3 \%\right)$ with the 
water flow (Figure 8) shows that the normalized Reynolds stress for the slurry is of about one half of that for water. A further decrease of the normalized Reynolds stress can be observed with the increasing concentration. The data for B134 show a slight difference between the Reynolds stress in the bottom part $(y / D=-0.5-0)$ and in the upper part $(y / D=0-0.5)$ of the cross section. This is probably caused by a presence of a concentration gradient.
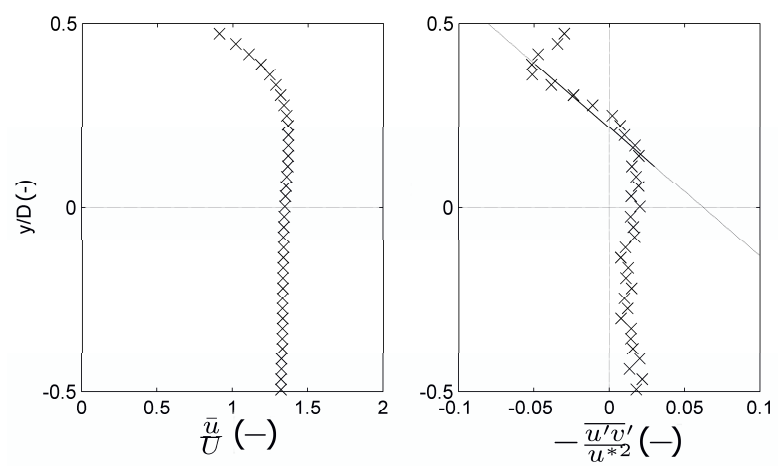

Figure 12. Ballotini B9 - distribution of normalized velocity (left) and Reynolds stress (right) for SF mode with delivered concentration of $24 \%$ and $U=1.7 \mathrm{~m} \cdot \mathrm{s}^{-1}\left(\operatorname{Re}=1.0 \cdot 10^{5}\right)$ using $2 \mathrm{MHz}$ transducer.
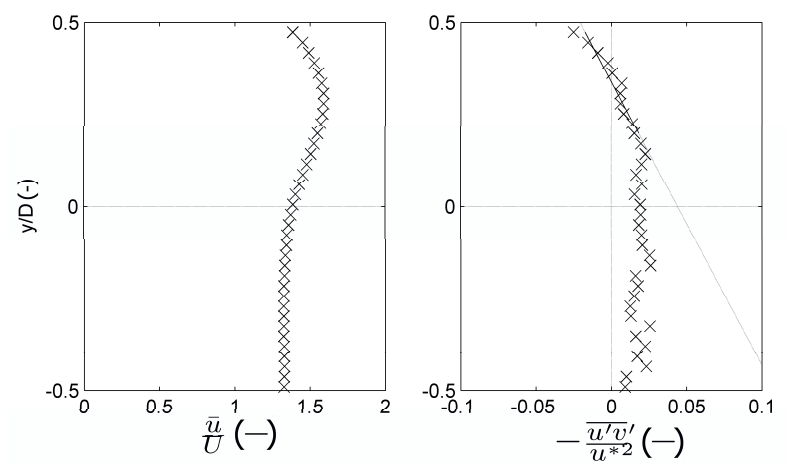

Figure 13. Ballotini B7 - distribution of normalized velocity (left) and Reynolds stress (right) for SF mode with delivered concentration of $24 \%$ and $U=1.7 \mathrm{~m} \cdot \mathrm{s}^{-1}\left(\operatorname{Re}=9.8 \cdot 10^{4}\right)$ using $1 \mathrm{MHz}$ transducer.

Experimental results for coarser grains are plotted in Figures 12 and 13 for the same velocity $\left(1.7 \mathrm{~m} \cdot \mathrm{s}^{-1}\right)$ and concentration (24\%) as in Figure 9. Due to the high settling velocity of the coarser grains, the HF mode was not reached even at higher velocities and the corresponding profiles differed only slightly from those in Figures 12 and 13. It can be clearly seen that in the lower part of the pipe cross section, the coarse-grain flow and the fine-grain flow behave very differently.

Measurements in this region cannot be correct for the coarse flows as the measured gradient of velocity is zero which is in contradiction with visual observations. The distribution of the Reynolds stress seems unrealistic as well. The values are unrealistically small in both the upper and the lower part of the profile. In the lower part, the shape of the distribution is unrealistic as well.

On the other hand, the zero shear stress corresponds well with the position of the maximum local velocity. This observation indicates that, at given concentration, the US beam penetrates roughly the first third of the pipe cross section height enabling relatively reliable measurement here. At lower positions, the signal is quickly damped as the local concentration increases. US signal attenuation is much more intense in slurries of two coarser fractions $(\mathrm{B} 7, \mathrm{~B} 9)$ than in the B134-slurry.

Figures 14 and 15 plot vertical distributions of the normalised Reynolds stress for B9 and B7, respectively. A comparison of the distributions for the constant delivered concentration of $\sim 10 \%$ (see crosses) in Figures 11,14 and 15 indicates that attenuation of measured fluctuations is increasing with the increasing grain size.

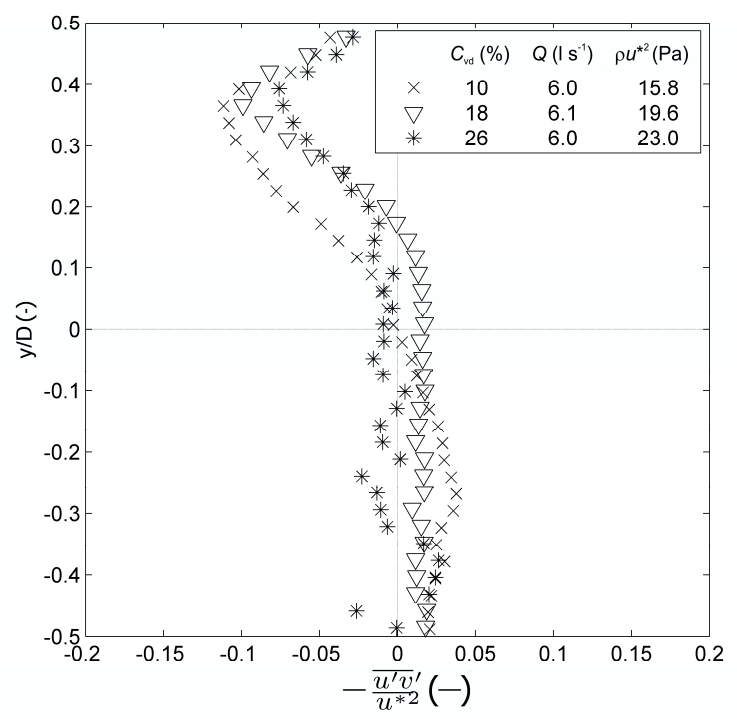

Figure 14. B9 - distribution of normalized Reynolds stress with delivered concentrations from 10 to $26 \%$ and $U \sim 2.3 \mathrm{~m} \cdot \mathrm{s}^{-1}$ (Re $\sim 1.5 \cdot 10^{5}$ ) using $2 \mathrm{MHz}$ transducers.

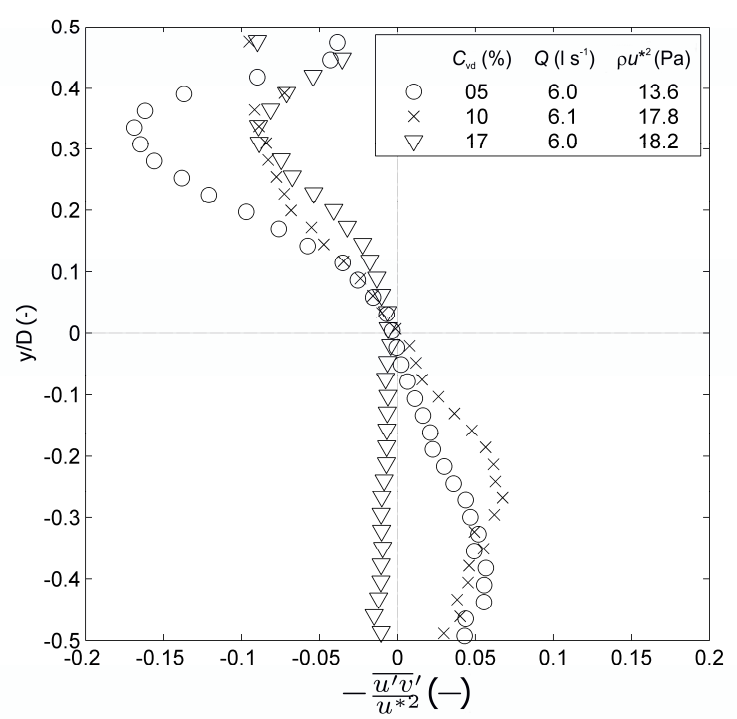

Figure 15. B134 - distribution of normalized Reynolds stress with delivered concentrations from 3 to $30 \%$ and $U \sim 2.3 \mathrm{~m} \cdot \mathrm{s}^{-1}$ $\left(\operatorname{Re} \sim 1.5 \cdot 10^{5}\right)$ using $2 \mathrm{MHz}$ transducer. 


\section{Discussions}

On one hand, the results of our experiments show the potential of the method of ultrasonic velocimetry to be used in Newtonian slurry flow carrying relatively coarse grains. One the other hand, the results show also limitations associated with the method.

Although the pulsed ultrasonic technique is applicable to various flow conditions, it requires a relatively large measurement volume. The measurement volume of the pulsed ultrasonic method has a disk-shape determined by the effective diameter of the piezoelectric element and the number and wave length of the wave cycles of the ultrasonic pulse. Kikura et al [17] reported that this became significant in an evaluation of the Reynolds stress distribution at lower basic frequencies of a transducer (1 or $2 \mathrm{MHz}$ ). However, a comparison of our results obtained in flow of clear water using the 2 and $4 \mathrm{MHz}$ transducers does not indicate any influence of frequency on the results (Figure 16).

Strong attenuation and multiscattering play a dominant role in two-phase flows. However, we seem to face a different problem. Since our grains are of considerable size, an employment of lower basic frequencies is an advantage.
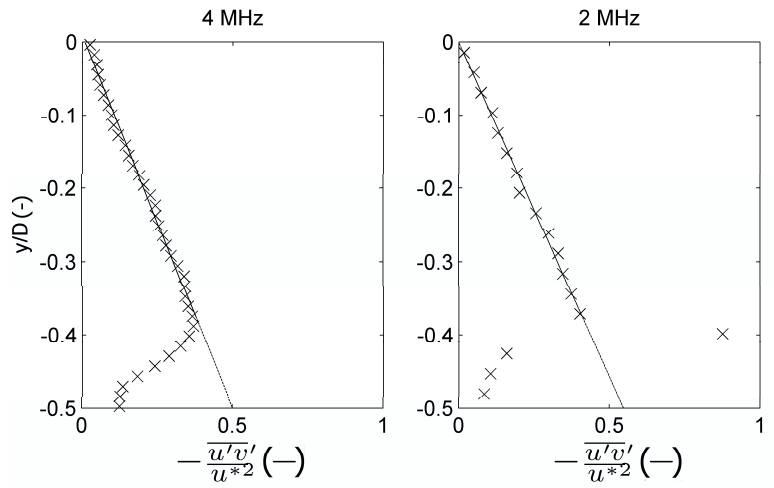

Figure 16. Distribution of Reynolds stress in lower half of height of flow of water $\left(\operatorname{Re} \sim 4.5 \cdot 10^{4}\right)$ - comparison of two different US transducers.

\section{Summary}

The study presents results of an ultrasonic velocimetry investigation of flows of water-ballotini slurries in a plexiglass pipe.

Reference measurements of turbulent flows of clear water provided expected distributions of longitudinal and vertical velocity components. However, measured levels of the normalized Reynolds stress seem to be significantly underestimated when compared to theoretical considerations.

The investigation of slurry flows brings information about the effect of the sediment transport regime on the velocity distribution in the pipe. Furthermore, the measurements reveal strong attenuation of velocity fluctuations caused by a presence of grains in the flow.

\section{Acknowledgments}

The research has been supported by the Czech Science Foundation through the grant project No. P105/12/1082 and by the Czech Technical University in Prague through the grant project No. SGS10/238/OHK1/3T/11.

\section{References}

1. M. Capper, T. Bruce, C. Gouble, Dynam. Atmos. Oceans, 31, 233-245 (2000)

2. H. Frey, M. Church, Science, 325, 1509-1510 (2009)

3. M. Chiari, K. Friedl, D. Rickenmann, J. Hydraul. Res., 48, 152-160 (2010)

4. D. Rickenmann, A. Koschni, Hydrol. Process., 24, 993-1007 (2010)

5. A. Recking, P. Frey, A. Paquier, P. Belleudy, J. Y. Champagne, J. Hydraul. Eng.-ASCE, 134, 1302$1310(2008)$

6. H. Capart, L. Fraccarollo: Transport layer structure in intense bed-load. Geophys. Res. Lett., 38, L20402, 6 p. (2011)

7. D. Berzi, J. Hydraul. Eng.-ASCE, 137, 1200-1207 (2011)

8. V. Matoušek, J. Hydraul. Eng.-ASCE, 135, 1101$1106(2009)$

9. V. Matoušek, J. Krupička, Proc. Second European IAHR Congress (Munich, Germany, 2012)

10. J. L. Best, A. D. Kirkbride, J. Peakall, Mean Flow and Turbulence Structure of Sediment-Laden Gravity Currents: New Insights using Ultrasonic Doppler Velocity Profiling. Particulate Gravity Currents, Ch. 12 (2009)

11. J. Wiklund, M. Stading, Flow Meas. Instrum., 19, 171-179 (2008)

12. B. Birkhofer, S. A. K. Jeelani, B. Ouriev, E. J. Windhab, In-Line Characterization and Rheometry of Concentrated Suspension Using Ultrasound (Proceedings - 4th ISUD, Sapporo, Japan), 65-68. (2004)

13. T. Song, W. H. Graf, J. Hydraul. Eng.-ASCE, 122, 141-154 (1996)

14. V. Bareš, Flow Meas. Instrum., 19, 145-154 (2008)

15. B. E. Launder, W. M. Ying, J. Fluid Mech., 54, 289295 (1972)

16. D. W. Knight, H. S. Patel, J. Hydraul. Eng.-ASCE, 111, 29-47 (1985)

17. H. Kikura, G. Yamanaka, M. Aritomi, Exp. Fluids, 36, 187-196 (2004) 